\title{
ASSESSMENT OF LIQUEFACTION POTENTIAL FOR SEISMIC RISK REDUCTION IN NORTH- EAST INDIA
}

\author{
Sangjukta Das, Tapati Parashar \\ Bachelor of Technology \\ Royal School of Engineering and Technology \\ Yudhajit Dey \\ Assistant Professor, \\ Royal School of Engineering and Technology
}

\begin{abstract}
The study of liquefaction potential of a region is of utmost importance regarding the safety of both life and property. Soil Liquefaction occurs when is there is loss of strength and stiffness in saturated and cohesion less soil due to increase in pore water pressure. The strength of the soil is sometimes reduced by earthquake shaking or rapid loading. Liquefaction causes soil failures which leads to severe damages to structures supported on such grounds leading to significant economic losses. The main purpose of the present study is to analyse liquefaction of some selected sites of Northeast India which falls in the zone of highest seismic risk zone level(Zone $\mathrm{V}$ in India)using bore log data of 95boreholes upto a depth of $15 \mathrm{~m}$. The liquefaction analysis is carried out with the help of 2 different methods and results are compared. Factor of safety versus depth curves plotted for showing the change in soil liquefaction with increasing depth. The methods used for analysis are simplified approach by Seed and Idriss (1971) and IS code procedure for evaluation of liquefaction potential (2016). More detailed study can be done in future and also various mitigation strategies can be put forward to reduce the impact of hazard.
\end{abstract}

Keywords- Borehole data, Cyclic resistance ratio, Cyclic stress ratio, Factor of safety, Liquefaction.

\section{INTRODUCTION}

Liquefaction is typically associated with earthquake-induced shaking that causes the ground to lose its bearing strength and act like a fluid. This can cause entire buildings to topple and cars to get sucked in. It can cause surface layers to slide downhill, damaging roads and rupturing distributed infrastructure systems like water and gas lines Earthquakes seismic event caused number of disturbances in the ground which could harm or damage structural stability leading to liquefaction. The construction of building near water bodies use retaining walls which are heavily dependent on the strength and stiffness of the soil. Once the soil gets liquefied, the retaining wall collapse which could cause landslide. The best known cases of foundation failures due to liquefaction are those that occurred during the 1964 earthquake in Niigata, Japan (Kishida, 1966).

Liquefaction is typically characterized by generation of excess pore pressure under undrained loading. The tendency of loose sands to densify under drained loading is well known. When loose sands are saturated and loaded under undrained conditions, the tendency to densify causes an increase in pore pressure, leading to a decrease in effective confining pressure. This lowers the shear strength of the soil, causing it to liquefy.

Guwahati city lies on a very high seismic zone (zone V) as per IS: 1893 along with the entire North Eastern region vulnerable to major earthquakes. The drifting of the Indian subcontinent also plate towards the Eurasian plate with the passage of time has been one of the sole reasons of earthquake occurrence in this part of the world. The northward moving Indian plate at the rate of $20 \mathrm{~mm} /$ year can provide earthquakes of magnitudes 8 and above every few hundred years. Most part of the city is built on soft sediments it can reasonably amplify the earthquake ground motion in case of a seismic event. Such areas have high risk of liquefaction occurrence of a strong intensity earthquake.

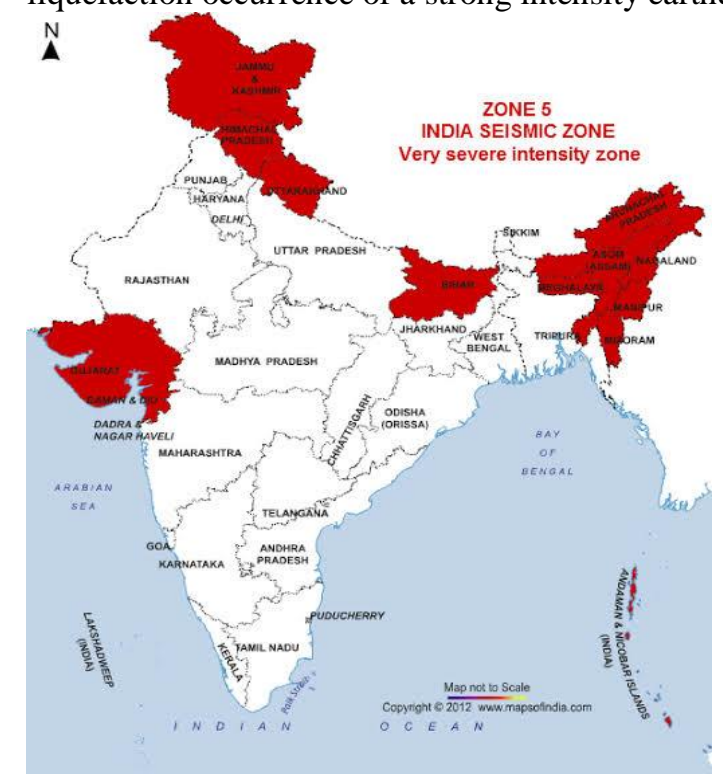


Fig1:- INDIA Seismic Zone

\section{BACKGROUND INFORMATION}

The devastating effect of liquefaction was first observed during various worldwide earthquakes including the 1987 great Assam Earthquake and the 1950 Assam earthquake both of magnitude 8.7 approximately. These instances of liquefaction necessitate the need to evaluate liquefaction susceptibility of an area. Evaluations of soil liquefaction potential using laboratory as well as several field techniques like standard penetration test (SPT), cone penetration test(CPT), shear wave velocity(Vs) etc. have become popular among the practising engineers.

Analysis of Guwahati city was performed by Nath et al. (2008) and PGA falls in the range $0.22 \mathrm{~g}-1.27 \mathrm{~g}$ with amplification factor 2-10. Different research developments in determination of liquefaction potential using the deterministic as well as the probabilistic approach have evolved due to the continued effort of many research workers. The developments started with the pioneering work of Seed and Idriss in 1971, and gradually by Seed and Idriss (1982) and Seed et al. (1985), by Liao and Whitman (1986), by Idriss (1999), NCEER Workshops- (1996 and 1997), by Toprak et al. (1999), by Juang et al. (2002), by Cetin et al. (2004), by Idriss and Boulanger $(2004,2006)$ etc.

A detailed study on the status of liquefaction potential of Guwahati city was first attempted by Raghukanth and Dash (2010) based on the scenario earthquakes of this region. Accordingto their research findings, the central part of the city near Dispur, Chandmari and along the Guwahati-Shillong (GS) road is highly vulnerable to liquefaction. The FOS was found to be less than 1 in almost all the places under study which means almost entire Guwahati city is susceptible to liquefaction.

\section{STUDY AREA}

Guwahati city which lies in the North Eastern region of India, falls in the highest seismic risk zonal level i.e. zone $\mathrm{V}$ in India. Liquefaction of soil and its associated damages have been widely observed in many previous earthquakes in North Eastern region of Assam. The entire North Eastern region has witnessed many high magnitude earthquakes in the past decades.

Guwahati, is India`s biggest city that falls in zone V. Guwahati is also amongst the list of cities, Government of India has shortlisted to be developed as "smart city". For this reason, the city attracts a lot of infrastructural growth in the times to come. Also recent developments have led to more construction of oil refineries, industries, hospitals, flyovers, multiplex halls etc. These heavy buildings require pile foundations which are embedded deep into the ground because of soil support. But if the soil is not strong enough to support then the foundation buckle which leads to collapsing of the structure. A major earthquake in this region shalllead to extensive damages to life and property.

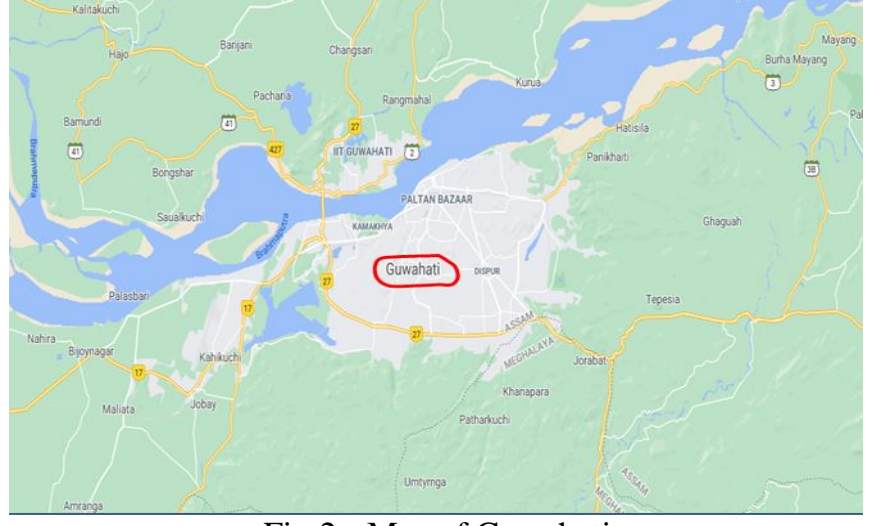

Fig 2:- Map of Guwahati

The evaluation was done by using 95bore log datas, which were collected from the different infrastructural, commercial and educational centres of Guwahati where the development is thick and fast with every passing year along with a rise in population. Hence it is important to check liquefaction so that adequate precaution can be taken before construction. The obtained values and results will provide very useful information to field engineers regarding where and who much ground improvement is required for avoiding future liquefaction. In addition, the present study will be helpful for planning and microzonation of the city.

\section{METHODOLOGY}

In order to analyse liquefaction of Guwahati city 95 boreholes of $15 \mathrm{~m}$ depth was taken The analysis for liquefaction was done for each depth of the bore hole using Simplified Approach Method by Seed and Idriss(1971) and IS Code Procedure for Evaluation of Liquefaction Potential (2016). Assumption of few data was taken accordingly.

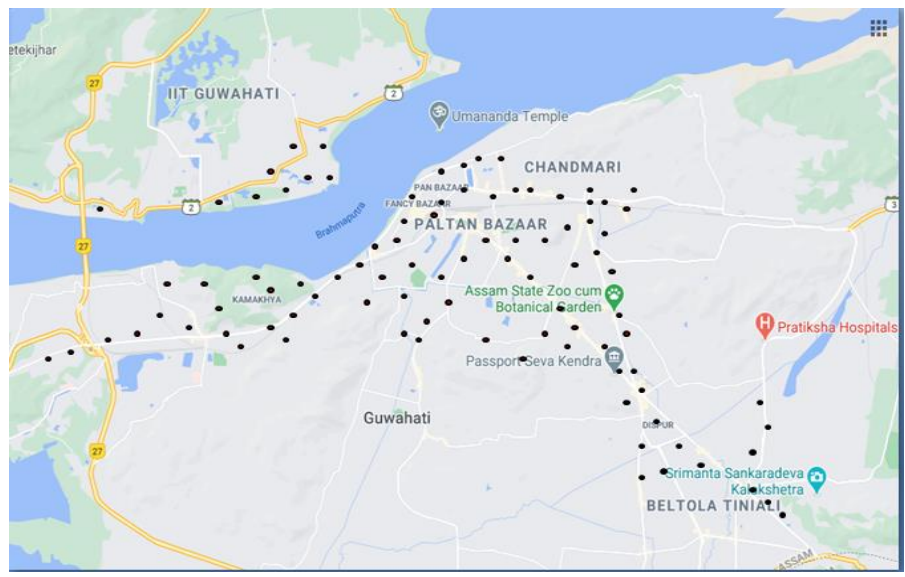

Fig3:- Location of the boreholes 
International Journal of Engineering Applied Sciences and Technology, 2020

Vol. 5, Issue 8, ISSN No. 2455-2143, Pages 219-228

Published Online December 2020 in IJEAST (http://www.ijeast.com)

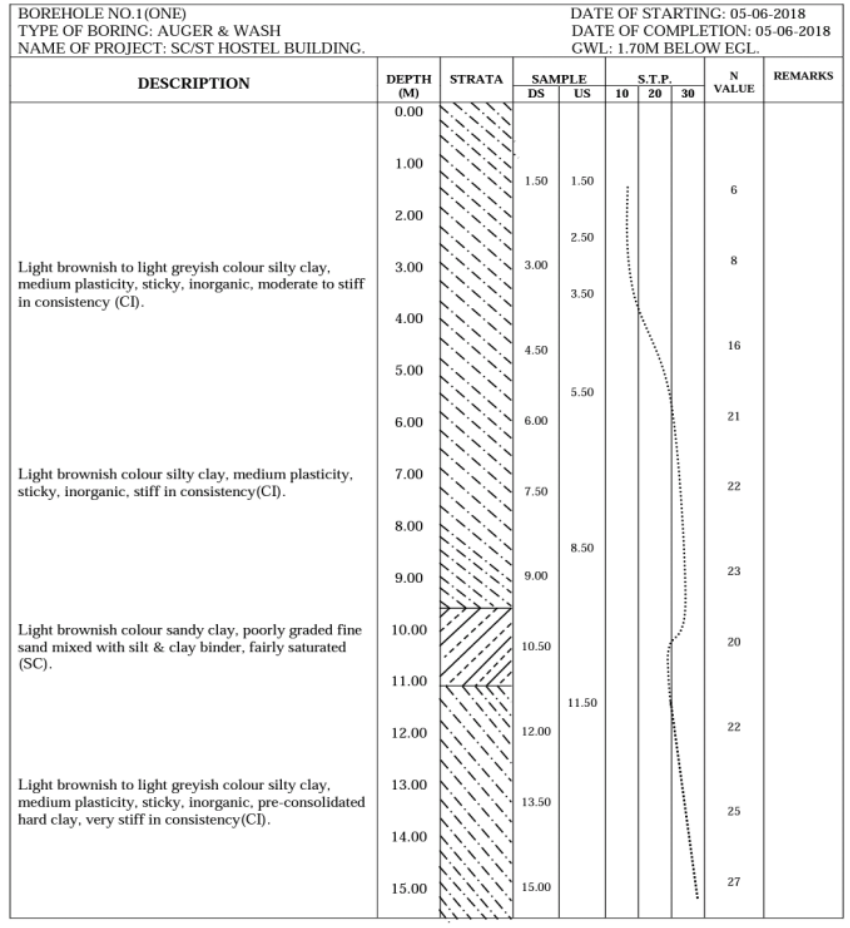

Fig4:-Collected borelog of one borehole.

\section{A.Procedure of Simplified Approach Method by Seed and} Idriss (1971)

The procedure allows the factor of safety (FOS) against liquefaction to be calculated for soil structure at a given depth.

With the help of specific gravity and void ratio, the saturated unit weight and submerged unit weight was found out by:-

$$
\begin{aligned}
& \gamma_{\text {sat }}=\left(\frac{\mathbf{g}+\mathbf{e}}{1+\mathbf{e}}\right) \gamma_{\mathrm{w}} \\
& \gamma_{\text {sub }}=\gamma_{\text {sat }}-\gamma_{w}
\end{aligned}
$$

Then total vertical stress $(\sigma \mathbf{v})$ and effective overburden pressure $\left(\sigma^{\prime} \mathbf{v}\right)$ was found out by:

$$
\begin{aligned}
& \sigma_{\mathrm{v} 1=\gamma_{\text {sat } \times} \mathrm{H}_{1}} \\
& \sigma_{\mathrm{v} 1=\gamma_{\text {sat }} \times \mathrm{H}_{1}}
\end{aligned}
$$

Then, value of stress reduction factor $\left(\mathbf{r}_{\mathbf{d}}\right)$ was calculated for depth H from Seed and Idriss curve(1971) shown in fig 3.

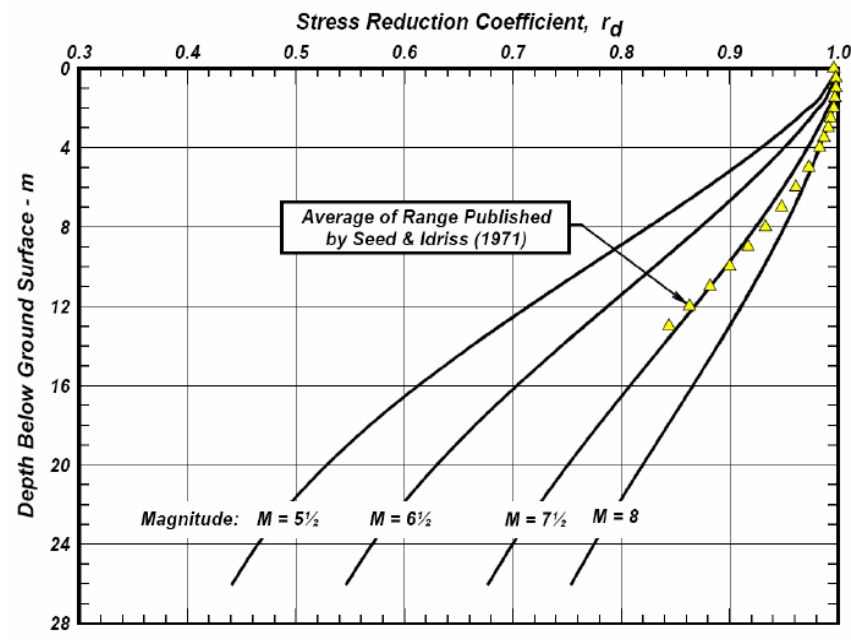

Fig5:- Seed and Idriss curve

Peak ground surface acceleration $\left(\mathbf{a}_{\max }\right)$ is considered as $0.36 \mathrm{~g}$.

Now, cyclic stress ratio(CSR) is given by:

$$
\operatorname{CSR}=0.65 \times \frac{a_{\max }}{g} \times \frac{\sigma_{v}}{\sigma_{v}^{\prime}} \times r_{d}
$$

Observed value of no. of blows $\left(\boldsymbol{N}_{\boldsymbol{m}}\right)$ is taken from bore $\log$ chart.

Now, overburden correction factor $\left(\mathbf{C}_{\mathbf{N}}\right)$ was calculated using the formula:

$$
C_{N}=9.81 \times \sqrt{\frac{1}{\sigma_{V}^{\prime}}}
$$

Actual hammer energy $\left(\boldsymbol{E}_{\boldsymbol{m}}\right)$ was considered to be $72 \%$ of theoretical free fall energy $\left(\boldsymbol{E}_{\text {eff }}\right)$.

Now, corrected value of no. of blows $\left(\boldsymbol{N}_{\mathbf{1}}\right)_{\mathbf{6 0}}$ was found out and hammer efficiency of $60 \%$ was taken:-

$$
\left(N_{1}\right)_{60}=N_{m} \times C_{N} \times \frac{E_{m}}{0.6 E_{\text {eff }}}
$$

Then, the value of CRR was taken from $\left(\boldsymbol{N}_{\mathbf{1}}\right)_{\mathbf{6 0}}$ versus CRR curve shown in Fig 4. 


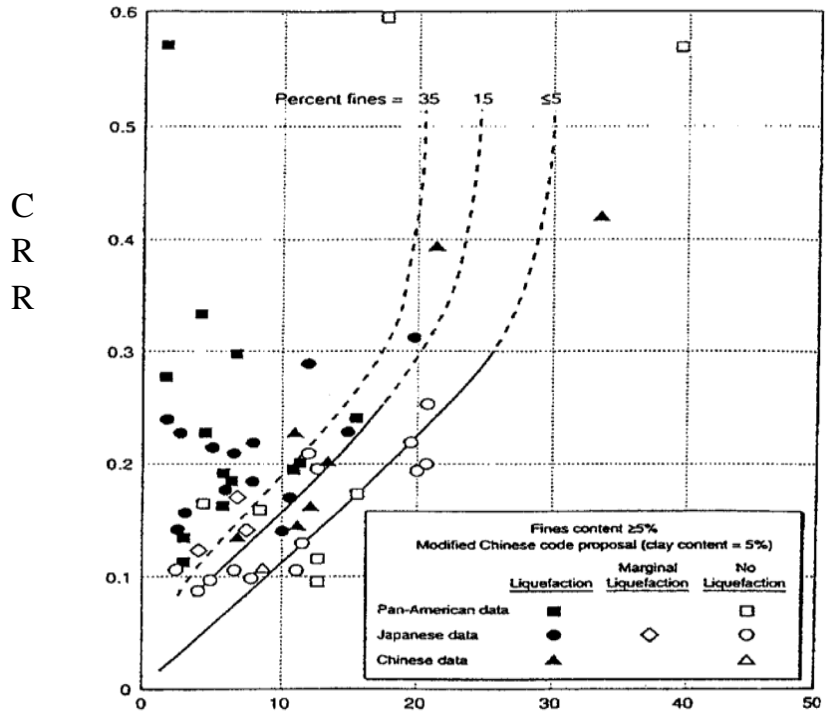

Standard Penetration Test Blow Count $\left(\mathrm{N}_{1}\right)_{60}$ Fig6:- $\left(N_{1}\right)_{60}$ vs. CRR curve

Finally, factor of safety was calculated by:-

$$
\text { FOS }=\frac{\text { CRR }}{\text { CSR }}
$$

If FOS is less than 1, soil will undergo liquefaction.

If FOS is more than 1, soil will not undergo liquefaction.

\section{B. Procedure of IS code Procedure for Evaluation of Liquefaction Potential (2016)}

The procedure allows the factor of safety (FOS) against liquefaction to be calculated for soil structure at a given depth.

With the help of specific gravity and void ratio, the saturated unit weight and submerged unit weight was found out by:-

$$
\begin{gathered}
\gamma_{\text {sat }}=\left(\frac{\mathbf{g}+\mathbf{e}}{1+\mathbf{e}}\right) \gamma_{\mathrm{w}} \\
\gamma_{\text {sub }}=\gamma_{\text {sat }}-\gamma_{\mathrm{w}}
\end{gathered}
$$

Then total vertical stress $\left(\sigma_{v}\right)$ and effective overburden $\operatorname{pressure}\left(\sigma_{\mathrm{v}}^{\prime}\right)$ was found out by:

$$
\begin{aligned}
& \sigma_{\mathrm{v} 1=\gamma_{\text {sat } \times} \mathrm{H}_{1}} \\
& \sigma_{\mathrm{v} 1=\gamma_{\text {sat } \times} \mathrm{H}_{1}}^{\prime}
\end{aligned}
$$

Then, value of stress reduction factor $\left(\mathbf{r}_{\mathbf{d}}\right)$ was calculated for depth $\mathrm{H}$ by:-

$$
1-(0.00765 \times \mathbf{H})
$$

Peak ground surface acceleration $\left(\mathbf{a}_{\max }\right)$ is considered as $0.36 \mathrm{~g}$.

Now, cyclic stress ratio (CSR) is given by:-

$$
\operatorname{CSR}=0.65 \times \frac{a_{\max }}{g} \times \frac{\sigma_{v}}{\sigma_{v}^{\prime}} \times r_{d}
$$

Observed value of no. of blows $\left(\mathbf{N}_{\mathbf{6 0}}\right)$ is taken from bore $\log$ chart.

Now, the value of $\left(\mathbf{N}_{\mathbf{1}}\right)_{\mathbf{6 0}}$ is calculated by:-

$$
\left(\mathbf{N}_{1}\right)_{60}=\mathbf{C}_{\mathrm{N}} \times \mathbf{N}_{60}
$$

Then, the value of CRR was found out from the $\left(N_{1}\right)_{60}$ vs. CRR curve.

Finally, factor of safety was calculated by:-

$$
\text { FOS }=\frac{\text { CRR }}{\text { CSR }}
$$

If FOS is less than 1, soil will undergo liquefaction. If FOS is more than 1, soil will not undergo liquefaction

Here, $\boldsymbol{\gamma}_{\mathbf{d}}=$ dry unit weight of soil in $\mathrm{KN} / \mathrm{m}^{3}, \boldsymbol{\gamma}_{\text {sat }}=$ saturated unit weight of soil in $\mathrm{KN} / \mathrm{m}^{3}, \boldsymbol{\gamma}_{\mathrm{w}}=$ unit weight of water in $\mathrm{KN} / \mathrm{m}^{3}, \mathbf{H}=$ depth of bore hole in $\mathrm{m}, \boldsymbol{\sigma}_{\mathbf{v}}=$ vertical stress in $\mathrm{KN} / \mathrm{m}^{2}, \boldsymbol{\sigma}^{\prime}{ }_{\mathbf{v}}=$ effective overburden pressure in $\mathrm{KN} / \mathrm{m}^{2}, \mathbf{a}_{\max }=$ peak ground surface acceleration, $\mathbf{g}=$ acceleration due to gravity, $\mathbf{r}_{\mathbf{d}}=$ value of stress reduction factor, $\mathbf{C S R}=$ cyclic stress ratio, $\mathbf{N}_{\mathbf{m}}=$ observed values of number of blows, $\mathbf{C}_{\mathbf{N}}=$ overburden correction factor, $\mathbf{E m}=$ actual hammer energy, $\boldsymbol{E}_{\text {eff }}=$ theoretical free fall energy, $\left(\boldsymbol{N}_{\mathbf{1}}\right)_{\mathbf{6 0}}=$ corrected values of number of blows normalised for $100 \mathrm{kPa}$ overburden pressure and hammer efficiency of $60 \%$., $\mathbf{C R R}=$ cyclic resistance ratio, $\mathbf{F O S}$ = factor of safety.

FOS is calculated by using the same method as above for all the depths for borehole data collected which is represented in the table below.

The factor of safety calculations of 8 boreholes out of 95 are shown below.

\section{A.BOREHOLE I}

\begin{tabular}{|c|c|c|l|c|c|c|c|c|c|}
\hline Depth & $\left.\sigma_{\mathrm{V}}^{\prime} \mathrm{kN} / \mathrm{m}^{2}\right)$ & $\left.\sigma_{\mathrm{V}} \mathrm{kN} / \mathrm{m}^{2}\right)$ & $\mathrm{r}_{\mathrm{d}}$ & $\mathrm{CSR}$ & $\mathrm{N}_{60}$ & $\left(N_{1}\right)_{60}$ & $\mathrm{CRR}$ & FOS & Liquefaction \\
\hline 1.5 & 28.74 & 28.74 & 0.988 & 0.231 & 6 & 11.22 & 0.19 & 0.82 & Yes \\
\hline 3 & 44.95 & 57.68 & 0.977 & 0.293 & 8 & 12.01 & 0.22 & 0.750 & Yes \\
\hline 4.5 & 59.38 & 86.81 & 0.965 & 0.330 & 16 & 20.90 & 0.5 & 1.51 & N0 \\
\hline 6 & 74 & 116.14 & 0.954 & 0.350 & 21 & 24.57 & 0.5 & 1.42 & N0 \\
\hline 7.5 & 88.7 & 145.5 & 0.942 & 0.361 & 22 & 23.51 & 0.5 & 1.381 & N0 \\
\hline 9 & 103.52 & 175.06 & 0.931 & 0.368 & 23 & 22.75 & 0.5 & 1.358 & N0 \\
\hline 10.5 & 118.55 & 204.79 & 0.919 & 0.372 & 20 & 18.48 & 0.21 & 0.566 & Yes \\
\hline 12 & 133.72 & 234.55 & 0.908 & 0.372 & 22 & 19.15 & 0.4 & 1.07 & N0 \\
\hline 13.5 & 148.74 & 264.31 & 0.896 & 0.372 & 25 & 20.63 & 0.5 & 1.344 & N0 \\
\hline 15 & 163.84 & 294.071 & 0.885 & 0.371 & 27 & 21.23 & 0.5 & 1.347 & N0 \\
\hline
\end{tabular}

Table1:-FOS by Seed and Idriss 
International Journal of Engineering Applied Sciences and Technology, 2020

Vol. 5, Issue 8, ISSN No. 2455-2143, Pages 219-228

Published Online December 2020 in IJEAST (http://www.ijeast.com)

\begin{tabular}{|c|c|c|c|c|c|c|c|c|c|}
\hline Depth & $\left.\sigma_{V}^{\prime} \mathrm{kN} / \mathrm{m}^{2}\right)$ & $\left.\sigma_{\mathrm{V}} \mathrm{kV} / \mathrm{m}^{2}\right)$ & $\mathrm{r}_{\mathrm{d}}$ & CSR & $\mathrm{N}_{60}$ & $\left(N_{1}\right)_{60}$ & CRR & F0S & Liquefaction \\
\hline 1.5 & 28.74 & 28.74 & 0.988 & 0.231 & 6 & 11.22 & 0.19 & 0.82 & Yes \\
\hline 3 & 44.95 & 57.68 & 0.977 & 0.293 & 8 & 12.01 & 0.22 & 0.750 & Yes \\
\hline 4.5 & 59.38 & 86.81 & 0.965 & 0.330 & 16 & 20.90 & 0.5 & 1.51 & $\mathrm{~N}_{0}$ \\
\hline 6 & 74 & 116.14 & 0.954 & 0.350 & 21 & 24.57 & 0.5 & 1.42 & $\mathrm{~N}_{0}$ \\
\hline 7.5 & 88.7 & 145.5 & 0.942 & 0.361 & 22 & 23.51 & 0.5 & 1.381 & $\mathrm{~N}_{0}$ \\
\hline 9 & 103.52 & 175.06 & 0.931 & 0.368 & 23 & 22.75 & 0.5 & 1.358 & $\mathrm{~N}_{0}$ \\
\hline 10.5 & 118.55 & 204.79 & 0.919 & 0.372 & 20 & 18.48 & 0.21 & 0.566 & Yes \\
\hline 12 & 133.72 & 234.55 & 0.908 & 0.372 & 22 & 19.15 & 0.4 & 1.07 & $\mathrm{~N}_{0}$ \\
\hline 13.5 & 148.74 & 264.31 & 0.896 & 0.372 & 25 & 20.63 & 0.5 & 1.344 & $\mathrm{~N}_{0}$ \\
\hline 15 & 163.84 & 294.071 & 0.885 & 0.371 & 27 & 21.23 & 0.5 & 1.347 & $\mathrm{~N}_{0}$ \\
\hline
\end{tabular}

Table2:-FOS by IS Code Procedure

B. BOREHOLE II

\begin{tabular}{|c|c|c|c|c|c|c|c|c|c|c|}
\hline Depth & $\left.\sigma_{\mathrm{V}}^{\prime} \mathrm{kN} / \mathrm{m}^{2}\right)$ & $\left.\sigma_{\mathrm{V}} \mathrm{kN} / \mathrm{m}^{2}\right)$ & $\mathrm{r}_{\mathrm{d}}$ & $\mathrm{CSR}$ & $\mathrm{N}_{\mathrm{m}}$ & $\mathrm{C}_{\mathrm{N}}$ & $\left(\mathrm{N}_{1}\right)_{60}$ & CRR & FOS & Liquefaction \\
\hline 1.5 & 29.04 & 29.04 & 0.998 & 0.234 & 3 & 1.82 & 6.552 & 0.12 & 0.512 & Yes \\
\hline 3 & 43.29 & 57.96 & 0.982 & 0.307 & 8 & 1.49 & 14.30 & 0.25 & 0.814 & Yes \\
\hline 4.5 & 57.67 & 87.07 & 0.954 & 0.337 & 11 & 1.29 & 17.028 & 0.29 & 0.860 & Yes \\
\hline 6 & 72.19 & 116.29 & 0.95 & 0.358 & 12 & 1.154 & 16.61 & 0.26 & 0.726 & Yes \\
\hline 7.5 & 86.82 & 145.62 & 0.94 & 0.368 & 14 & 1.052 & 17.67 & 0.32 & 0.869 & Yes \\
\hline 9 & 101.61 & 175.11 & 0.91 & 0.366 & 15 & 0.97 & 17.48 & 0.31 & 0.846 & Yes \\
\hline 10.5 & 116.44 & 204.64 & 0.89 & 0.366 & 18 & 0.909 & 19.63 & 0.39 & 1.065 & $\mathrm{~N}_{0}$ \\
\hline 12 & 131.28 & 234.18 & 0.85 & 0.354 & 21 & 0.856 & 21.57 & 0.5 & 1.41 & $\mathrm{~N}_{0}$ \\
\hline 13.5 & 146.145 & 263.74 & 0.825 & 0.348 & 20 & 0.811 & 19.46 & 0.38 & 1.091 & $\mathrm{~N}_{0}$ \\
\hline 15 & 161.01 & 293.31 & 0.754 & 0.321 & 23 & 0.773 & 21.33 & 0.5 & 1.55 & $\mathrm{~N}_{0}$ \\
\hline
\end{tabular}

Table3:-FOS by Seed and Idriss

\begin{tabular}{|c|c|c|c|c|c|c|c|c|c|}
\hline Depth & $\left.\sigma_{V}^{\prime} \mathrm{kV} / \mathrm{m}^{2}\right)$ & $\left.\sigma_{V} \mathrm{kV} / \mathrm{m}^{2}\right)$ & $\mathrm{r}_{\mathrm{d}}$ & CSR & $\mathrm{N}_{60}$ & $\left(N_{1}\right)_{60}$ & CRR & FOS & Liquefaction \\
\hline 1.5 & 29.04 & 29.04 & 0.988 & 0.231 & 3 & 5.60 & 0.11 & 0.47 & Yes \\
\hline 3 & 43.29 & 57.96 & 0.977 & 0.306 & 8 & 12.23 & 0.23 & 0.751 & Yes \\
\hline 4.5 & 57.67 & 87.07 & 0.965 & 0.340 & 11 & 14.58 & 0.25 & 0.735 & Yes \\
\hline 6 & 72.19 & 116.29 & 0.954 & 0.359 & 12 & 14.21 & 0.249 & 0.69 & Yes \\
\hline 7.5 & 86.82 & 145.62 & 0.942 & 0.369 & 14 & 15.12 & 0.26 & 0.704 & Yes \\
\hline 9 & 101.61 & 175.11 & 0.931 & 0.354 & 15 & 14.97 & 0.258 & 0.728 & Yes \\
\hline 10.5 & 116.44 & 204.64 & 0.919 & 0.349 & 18 & 16.79 & 0.35 & 1.002 & No \\
\hline 12 & 131.28 & 234.18 & 0.908 & 0.379 & 21 & 18.44 & 0.39 & 1.029 & No \\
\hline 13.5 & 146.145 & 263.74 & 0.896 & 0.320 & 20 & 16.65 & 0.32 & 1.00 & No \\
\hline 15 & 161.01 & 293.31 & 0.885 & 0.377 & 23 & 18.24 & 0.38 & 1.007 & No \\
\hline
\end{tabular}

Table4:-FOS by IS Code Procedure

C. BOREHOLE III

Table5:-FOS by Seed and Idriss

\begin{tabular}{|c|c|c|c|c|c|c|c|c|c|c|}
\hline Depth & $\left.\sigma_{\mathrm{V}}^{\prime} \mathrm{kN} / \mathrm{m}^{2}\right)$ & $\left.\sigma_{\mathrm{V}} \mathrm{kN} / \mathrm{m}^{2}\right)$ & $\mathrm{r}_{\mathrm{d}}$ & $\mathrm{CSR}$ & $\mathrm{N}_{\mathrm{m}}$ & $\mathrm{C}_{\mathrm{V}}$ & $\left(N_{1}\right)_{60}$ & $\mathrm{CRR}$ & FOS & Liquefaction \\
\hline 1.5 & 29.13 & 29.13 & 0.998 & 0.233 & 5 & 1.81 & 10.86 & 0.18 & 0.772 & Yes \\
\hline 3 & 43.41 & 58.11 & 0.982 & 0.307 & 6 & 1.48 & 10.65 & 0.19 & 0.618 & Yes \\
\hline 4.5 & 57.82 & 87.22 & 0.954 & 0.336 & 8 & 1.290 & 12.38 & 0.22 & 0.654 & Yes \\
\hline 6 & 72.36 & 116.46 & 0.95 & 0.357 & 11 & 1.15 & 15.18 & 0.25 & 0.700 & Yes \\
\hline 7.5 & 87.07 & 145.87 & 0.94 & 0.368 & 13 & 1.051 & 16.39 & 0.29 & 0.788 & Yes \\
\hline 9 & 101.88 & 175.98 & 0.91 & 0.367 & 14 & 0.971 & 16.31 & 0.285 & 0.776 & Yes \\
\hline 10.5 & 116.77 & 204.97 & 0.89 & 0.365 & 17 & 0.970 & 19.78 & 0.34 & 0.931 & Yes \\
\hline 12 & 131.745 & 234.64 & 0.85 & 0.354 & 16 & 0.854 & 16.39 & 0.29 & 0.819 & Yes \\
\hline 13.5 & 146.85 & 263.45 & 0.825 & 0.347 & 19 & 0.809 & 18.44 & 0.34 & 0.97 & Yes \\
\hline 15 & 161.98 & 294.28 & 0.754 & 0.320 & 18 & 0.770 & 16.63 & 0.30 & 0.937 & Yes \\
\hline
\end{tabular}

\begin{tabular}{|c|c|c|c|c|c|c|c|c|c|}
\hline Depth & $\left.\sigma_{V}^{\prime} \mathrm{kN} / \mathrm{m}^{2}\right)$ & $\left.\sigma_{V} \mathrm{kN} / \mathrm{m}^{2}\right)$ & $\mathrm{r}_{\mathrm{d}}$ & CSR & $\mathrm{N}_{60}$ & $\left(N_{1}\right)_{60}$ & CRR & F0S & Liquefaction \\
\hline 1.5 & 29.13 & 29.13 & 0.988 & 0.231 & 5 & 9.32 & 0.14 & 0.606 & Yes \\
\hline 3 & 43.41 & 58.11 & 0.977 & 0.306 & 6 & 9.16 & 0.18 & 0.184 & Yes \\
\hline 4.5 & 57.82 & 87.22 & 0.965 & 0.340 & 8 & 10.59 & 0.19 & 0.196 & Yes \\
\hline 6 & 72.36 & 116.46 & 0.954 & 0.359 & 11 & 13.01 & 0.21 & 0.220 & Yes \\
\hline 7.5 & 87.07 & 145.87 & 0.942 & 0.369 & 13 & 14.02 & 0.23 & 0.244 & Yes \\
\hline 9 & 101.88 & 175.98 & 0.931 & 0.376 & 14 & 13.69 & 0.22 & 0.236 & Yes \\
\hline 10.5 & 116.77 & 204.97 & 0.919 & 0.378 & 17 & 15.88 & 0.27 & 0.293 & Yes \\
\hline 12 & 131.745 & 234.64 & 0.908 & 0.378 & 16 & 14.031 & 0.23 & 0.253 & Yes \\
\hline 13.5 & 146.85 & 263.45 & 0.896 & 0.376 & 19 & 15.78 & 0.18 & 0.20 & Yes \\
\hline 15 & 161.98 & 294.28 & 0.885 & 0.376 & 18 & 14.23 & 0.15 & 0.16 & Yes \\
\hline
\end{tabular}

Table6:-FOS by IS Code Procedure 


\section{BOREHOLE IV}

\begin{tabular}{|c|c|c|c|c|c|c|c|c|c|c|}
\hline Depth & $\left.\sigma_{V}^{\prime} \mathrm{kN} / \mathrm{m}^{2}\right)$ & $\left.\sigma_{V} \mathrm{kN} / \mathrm{m}^{2}\right)$ & $\mathrm{r}_{\mathrm{d}}$ & $\mathrm{CSR}$ & $\mathrm{N}_{\mathrm{m}}$ & $\mathrm{C}_{\mathrm{N}}$ & $\left(N_{1}\right)_{60}$ & CRR & FOS & Liquefaction \\
\hline 1.5 & 28.53 & 28.53 & 0.998 & 0.233 & 6 & 1.836 & 13.21 & 0.22 & 0.94 & Yes \\
\hline 3 & 42.75 & 57.45 & 0.982 & 0.310 & 10 & 1.500 & 18 & 0.36 & 1.16 & No $_{0}$ \\
\hline 4.5 & 57.54 & 86.94 & 0.954 & 0.337 & 11 & 1.293 & 17.06 & 0.27 & 0.801 & Yes \\
\hline 6 & 72.28 & 116.38 & 0.95 & 0.357 & 12 & 1.152 & 16.58 & 0.28 & 0.78 & Yes \\
\hline 7.5 & 87.15 & 145.95 & 0.94 & 0.368 & 13 & 1.049 & 16.36 & 0.25 & 0.67 & Yes \\
\hline 9 & 102.16 & 175.66 & 0.91 & 0.366 & 13 & 0.960 & 14.97 & 0.24 & 0.65 & Yes \\
\hline 10.5 & 117.22 & 205.42 & 0.89 & 0.364 & 15 & 0.900 & 16.20 & 0.28 & 0.76 & Yes \\
\hline 12 & 132.28 & 235.18 & 0.85 & 0.353 & 17 & 0.850 & 17.34 & 0.34 & 1.01 & No \\
\hline 13.5 & 147.36 & 264.96 & 0.825 & 0.347 & 18 & 0.800 & 17.28 & 0.31 & 0.89 & Yes \\
\hline 15 & 162.43 & 294.73 & 0.754 & 0.320 & 19 & 0.760 & 17.32 & 0.33 & 1.03 & No \\
\hline
\end{tabular}

Table7:-FOS by Seed and Idriss

\begin{tabular}{|c|c|c|c|c|c|c|c|c|c|}
\hline Depth & $\left.\sigma_{\mathrm{V}}^{\prime} \mathrm{kN} / \mathrm{m}^{2}\right)$ & $\left.\sigma_{\mathrm{V}} \mathrm{kN} / \mathrm{m}^{2}\right)$ & $\mathrm{r}_{\mathrm{d}}$ & CSR & $\mathrm{N}_{60}$ & $\left(N_{1}\right)_{60}$ & CRR & FOS & Liquefaction \\
\hline 1.5 & 28.53 & 28.53 & 0.988 & 0.231 & 6 & 11.30 & 0.17 & 0.73 & Yes \\
\hline 3 & 42.75 & 57.45 & 0.977 & 0.307 & 10 & 15.39 & 0.33 & 1.07 & $\mathrm{~N}_{0}$ \\
\hline 4.5 & 57.54 & 86.94 & 0.965 & 0.341 & 11 & 14.59 & 0.19 & 0.5 & Yes \\
\hline 6 & 72.28 & 116.38 & 0.954 & 0.359 & 12 & 14.20 & 0.14 & 0.38 & Yes \\
\hline 7.5 & 87.15 & 145.95 & 0.942 & 0.369 & 13 & 14.01 & 0.23 & 0.62 & Yes \\
\hline 9 & 102.16 & 175.66 & 0.931 & 0.374 & 13 & 12.94 & 0.21 & 0.56 & Yes \\
\hline 10.5 & 117.22 & 205.42 & 0.919 & 0.376 & 15 & 13.94 & 0.23 & 0.61 & Yes \\
\hline 12 & 132.28 & 235.18 & 0.908 & 0.340 & 17 & 14.87 & 0.34 & 1.00 & No \\
\hline 13.5 & 147.36 & 264.96 & 0.896 & 0.376 & 18 & 14.92 & 0.34 & 0.64 & Yes \\
\hline 15 & 162.43 & 294.73 & 0.885 & 0.354 & 19 & 15.01 & 0.35 & 1.01 & No \\
\hline
\end{tabular}

Table8:-FOS by IS Code Procedure

E. BOREHOLE V

\begin{tabular}{|c|c|c|c|c|c|c|c|c|c|c|}
\hline Depth & $\left.\sigma_{V}^{\prime} \mathrm{kN} / \mathrm{m}^{2}\right)$ & $\left.\sigma_{\mathrm{V}} \mathrm{k} / \mathrm{m}^{2}\right)$ & $\mathrm{r}_{\mathrm{d}}$ & $\mathrm{CSR}$ & $\mathrm{N}_{\mathrm{m}}$ & $\mathrm{C}_{\mathrm{N}}$ & $\left(N_{1}\right)_{60}$ & CRR & F0S & Liquefaction \\
\hline 1.5 & 28.57 & 28.53 & 0.998 & 0.233 & 6 & 1.83 & 13.17 & 0.21 & 0.901 & Yes \\
\hline 3 & 42.74 & 57.45 & 0.982 & 0.308 & 11 & 1.499 & 19.78 & 0.39 & 1.266 & No \\
\hline 4.5 & 57.41 & 86.82 & 0.954 & 0.337 & 30 & 1.294 & 46.5 & 0.5 & 1.48 & No $_{0}$ \\
\hline 6 & 72.17 & 116.28 & 0.95 & 0.358 & 13 & 1.154 & 18.00 & 0.28 & 0.78 & Yes \\
\hline 7.5 & 87.01 & 145.81 & 0.94 & 0.368 & 12 & 1.051 & 15.13 & 0.26 & 0.706 & Yes \\
\hline 9 & 101.96 & 175.47 & 0.91 & 0.366 & 15 & 0.971 & 17.47 & 0.29 & 0.792 & Yes \\
\hline 10.5 & 117.02 & 205.23 & 0.89 & 0.365 & 19 & 0.906 & 20.65 & 0.45 & 1.23 & No \\
\hline 12 & 132.13 & 235.03 & 0.85 & 0.353 & 20 & 0.853 & 20.47 & 0.49 & 1.38 & No \\
\hline 13.5 & 147.26 & 264.87 & 0.825 & 0.347 & 18 & 0.808 & 17.45 & 0.28 & 0.80 & Yes \\
\hline 15 & 162.50 & 294.81 & 0.754 & 0.320 & 20 & 0.769 & 18.45 & 0.29 & 0.377 & Yes \\
\hline
\end{tabular}

Table9:-FOS by Seed and Idriss

\begin{tabular}{|c|c|c|c|c|c|c|c|c|c|}
\hline Depth & $\left.\sigma_{V}^{\prime} \mathrm{kN} / \mathrm{m}^{2}\right)$ & $\left.\sigma_{V} \mathrm{kN} / \mathrm{m}^{2}\right)$ & $\mathrm{r}_{d}$ & CSR & $\mathrm{N}_{60}$ & $\left(N_{1}\right)_{60}$ & CRR & FOS & Liquefaction \\
\hline 1.5 & 28.57 & 28.53 & 0.988 & 0.231 & 6 & 11.29 & 0.18 & 0.77 & Yes \\
\hline 3 & 42.74 & 57.45 & 0.977 & 0.307 & 11 & 16.93 & 0.33 & 1.07 & $\mathrm{~N}_{0}$ \\
\hline 4.5 & 57.41 & 86.82 & 0.965 & 0.341 & 30 & 39.85 & 0.5 & 1.46 & $\mathrm{~N}_{0}$ \\
\hline 6 & 72.17 & 116.28 & 0.954 & 0.359 & 13 & 15.40 & 0.22 & 0.61 & Yes \\
\hline 7.5 & 87.01 & 145.81 & 0.942 & 0.369 & 12 & 12.94 & 0.23 & 0.62 & Yes \\
\hline 9 & 101.96 & 175.47 & 0.931 & 0.374 & 15 & 14.95 & 0.24 & 0.64 & Yes \\
\hline 10.5 & 117.02 & 205.23 & 0.919 & 0.327 & 19 & 17.67 & 0.35 & 1.07 & N0 \\
\hline 12 & 132.13 & 235.03 & 0.908 & 0.327 & 20 & 17.51 & 0.33 & 1.009 & No \\
\hline 13.5 & 147.26 & 264.87 & 0.896 & 0.376 & 18 & 14.93 & 0.24 & 0.63 & Yes \\
\hline 15 & 162.50 & 294.81 & 0.885 & 0.375 & 20 & 15.79 & 0.33 & 0.81 & Yes \\
\hline
\end{tabular}

Table10:-FOS by IS Code Procedure

\section{F. BOREHOLE VI}

\begin{tabular}{|c|c|c|c|c|c|c|c|c|c|c|}
\hline Depth & $\left.\sigma_{\mathrm{V}}^{\prime} \mathrm{kN} / \mathrm{m}^{2}\right)$ & $\left.\sigma_{\mathrm{V}} \mathrm{kN} / \mathrm{m}^{2}\right)$ & $\mathrm{r}_{\mathrm{d}}$ & CSR & $\mathrm{N}_{\mathrm{m}}$ & $\mathrm{C}_{\mathrm{N}}$ & $\left(N_{1}\right)_{60}$ & CRR & FOS & Liquefaction \\
\hline 1.5 & 28.96 & 28.96 & 0.998 & 0.233 & 2 & 1.82 & 4.36 & 0.08 & 0.34 & Yes \\
\hline 3 & 43.37 & 58.07 & 0.982 & 0.307 & 7 & 1.48 & 12.43 & 0.18 & 0.58 & Yes \\
\hline 4.5 & 57.94 & 87.34 & 0.954 & 0.336 & 9 & 1.28 & 13.82 & 0.24 & 0.71 & Yes \\
\hline 6 & 72.61 & 116.71 & 0.95 & 0.355 & 11 & 1.15 & 15.04 & 0.28 & 0.78 & Yes \\
\hline 7.5 & 87.41 & 146.21 & 0.94 & 0.367 & 13 & 1.04 & 16.22 & 0.3 & 0.81 & Yes \\
\hline 9 & 102.24 & 175.79 & 0.91 & 0.366 & 10 & 0.97 & 11.64 & 0.21 & 0.57 & Yes \\
\hline 10.5 & 117.21 & 205.42 & 0.89 & 0.364 & 11 & 0.906 & 11.95 & 0.22 & 0.60 & Yes \\
\hline 12 & 132.16 & 235.06 & 0.85 & 0.353 & 14 & 0.853 & 14.33 & 0.25 & 0.70 & Yes \\
\hline 13.5 & 147.11 & 264.71 & 0.825 & 0.347 & 15 & 0.80 & 14.4 & 0.26 & 0.74 & Yes \\
\hline 15 & 162.07 & 294.38 & 0.754 & 0.320 & 16 & 0.77 & 14.78 & 0.27 & 0.84 & Yes \\
\hline
\end{tabular}

Table11:-FOS by Seed and Idriss 


\begin{tabular}{|c|c|c|c|c|c|c|c|c|c|}
\hline Depth & $\left.\sigma_{\mathrm{V}}^{\prime} \mathrm{kN} / \mathrm{m}^{2}\right)$ & $\left.\sigma_{\mathrm{V}} \mathrm{kN} / \mathrm{m}^{2}\right)$ & $\mathrm{r}_{\mathrm{d}}$ & $\mathrm{CSR}$ & $\mathrm{N}_{60}$ & $\left(\mathrm{~N}_{1}\right)_{60}$ & CRR & FOS & Liquefaction \\
\hline 1.5 & 28.96 & 28.96 & 0.988 & 0.231 & 2 & 3.74 & 0.08 & 0.34 & Yes \\
\hline 3 & 43.37 & 58.07 & 0.977 & 0.306 & 7 & 10.69 & 0.16 & 0.52 & Yes \\
\hline 4.5 & 57.94 & 87.34 & 0.965 & 0.340 & 9 & 11.90 & 0.21 & 0.61 & Yes \\
\hline 6 & 72.61 & 116.71 & 0.954 & 0.358 & 11 & 12.99 & 0.23 & 0.64 & Yes \\
\hline 7.5 & 87.41 & 146.21 & 0.942 & 0.315 & 13 & 13.99 & 0.25 & 0.79 & Yes \\
\hline 9 & 102.24 & 175.79 & 0.931 & 0.374 & 10 & 9.95 & 0.18 & 0.48 & Yes \\
\hline 10.5 & 117.21 & 205.42 & 0.919 & 0.376 & 11 & 10.22 & 0.2 & 0.53 & Yes \\
\hline 12 & 132.16 & 235.06 & 0.908 & 0.377 & 14 & 12.25 & 0.22 & 0.58 & Yes \\
\hline 13.5 & 147.11 & 264.71 & 0.896 & 0.377 & 15 & 12.44 & 0.22 & 0.58 & Yes \\
\hline 15 & 162.07 & 294.38 & 0.885 & 0.376 & 16 & 10.00 & 0.19 & 0.50 & Yes \\
\hline
\end{tabular}

Table12:-FOS by IS Code Procedure

\section{G. BOREHOLE VII}

\begin{tabular}{|c|c|c|c|c|c|c|c|c|c|c|}
\hline Depth & $\left.\sigma_{\mathrm{V}}^{\prime} \mathrm{kN} / \mathrm{m}^{2}\right)$ & $\left.\sigma_{\mathrm{V}} \mathrm{kN} / \mathrm{m}^{2}\right)$ & $\mathrm{r}_{\mathrm{d}}$ & $\mathrm{CSR}$ & $\mathrm{N}_{\mathrm{m}}$ & $\mathrm{C}_{\mathrm{N}}$ & $\left(\mathrm{N}_{1}\right)_{60}$ & CRR & FOS & Liquefaction \\
\hline 1.5 & 28.7 & 28.7 & 0.998 & 0.233 & 2 & 1.831 & 4.39 & 0.12 & 0.51 & Yes \\
\hline 3 & 42.09 & 56.83 & 0.982 & 0.31 & 2 & 1.512 & 3.62 & 0.1 & 0.32 & Yes \\
\hline 4.5 & 55.37 & 84.77 & 0.954 & 0.341 & 3 & 1.318 & 4.74 & 0.13 & 0.38 & Yes \\
\hline 6 & 69.06 & 113.16 & 0.95 & 0.422 & 4 & 1.180 & 5.66 & 0.14 & 0.33 & Yes \\
\hline 7.5 & 83.27 & 142.07 & 0.94 & 0.357 & 10 & 1.075 & 12.9 & 0.23 & 0.61 & Yes \\
\hline 9 & 97.50 & 171.0 & 0.91 & 0.373 & 15 & 0.99 & 17.8 & 0.29 & 0.77 & Yes \\
\hline 10.5 & 111.42 & 199.62 & 0.89 & 0.373 & 10 & 0.929 & 11.14 & 0.21 & 0.56 & Yes \\
\hline 12 & 125.57 & 228.47 & 0.85 & 0.361 & 20 & 0.875 & 21 & 0.45 & 1.24 & No \\
\hline 13.5 & 139.25 & 256.85 & 0.825 & 0.356 & 19 & 0.831 & 18.9 & 0.4 & 1.12 & No \\
\hline 15 & 152.78 & 285.08 & 0.754 & 0.329 & 13 & 0.793 & 12.3 & 0.22 & 0.66 & Yes \\
\hline
\end{tabular}

Table13:-FOS by Seed and Idriss

\begin{tabular}{|c|c|c|c|c|c|c|c|c|c|}
\hline Depth & $\left.\sigma_{V}^{\prime} \mathrm{kN} / \mathrm{m}^{2}\right)$ & $\left.\sigma_{\mathrm{V}} \mathrm{kN} / \mathrm{m}^{2}\right)$ & $\mathrm{r}_{\mathrm{d}}$ & CSR & $\mathrm{N}_{60}$ & $\left(\mathrm{~N}_{1}\right)_{60}$ & CRR & FOS & Liquefaction \\
\hline 1.5 & 28.7 & 28.7 & 0.988 & 0.231 & 2 & 11.2 & 0.2 & 0.86 & Yes \\
\hline 3 & 42.09 & 56.83 & 0.977 & 0.30 & 2 & 3.103 & 0.09 & 0.3 & Yes \\
\hline 4.5 & 55.37 & 84.77 & 0.965 & 0.345 & 3 & 4.05 & 0.12 & 0.34 & Yes \\
\hline 6 & 69.06 & 113.16 & 0.954 & 0.365 & 4 & 4.84 & 0.13 & 0.35 & Yes \\
\hline 7.5 & 83.27 & 142.07 & 0.942 & 0.376 & 10 & 11.03 & 0.19 & 0.50 & Yes \\
\hline 9 & 97.50 & 171.0 & 0.931 & 0.382 & 15 & 15.2 & 0.25 & 0.65 & Yes \\
\hline 10.5 & 111.42 & 199.62 & 0.919 & 0.385 & 10 & 9.41 & 0.14 & 0.36 & Yes \\
\hline 12 & 125.57 & 228.47 & 0.908 & 0.386 & 20 & 17.96 & 0.39 & 1.01 & No \\
\hline 13.5 & 139.25 & 256.85 & 0.896 & 0.386 & 19 & 16.2 & 0.389 & 1.00 & No \\
\hline 15 & 152.78 & 285.08 & 0.885 & 0.436 & 13 & 10.5 & 0.18 & 0.41 & Yes \\
\hline
\end{tabular}

Table14:-FOS by IS Code Procedure
H. BOREHOLE VIII

\begin{tabular}{|c|c|c|c|c|c|c|c|c|c|c|}
\hline Depth & $\left.\sigma_{V}^{\prime} \mathrm{kN} / \mathrm{m}^{2}\right)$ & $\left.\sigma_{\mathrm{V}} \mathrm{kN} / \mathrm{m}^{2}\right)$ & $\mathrm{r}_{\mathrm{d}}$ & $\mathrm{CSR}$ & $\mathrm{N}_{\mathrm{m}}$ & $\mathrm{C}_{\mathrm{N}}$ & $\left(N_{1}\right)_{60}$ & $\mathrm{CRR}$ & F0S & Liquefaction \\
\hline 1.5 & 28.8 & 28.8 & 0.998 & 0.231 & 3 & 1.827 & 6.57 & 0.13 & 0.56 & Yes \\
\hline 3 & 57.39 & 86.82 & 0.982 & 0.347 & 5 & 1.29 & 7.76 & 0.16 & 0.46 & Yes \\
\hline 4.5 & 100.68 & 174.25 & 0.954 & 0.389 & 8 & 0.997 & 9.37 & 0.18 & 0.46 & Yes \\
\hline 6 & 159.3 & 291.73 & 0.95 & 0.405 & 12 & 0.977 & 14.06 & 0.24 & 0.59 & Yes \\
\hline 7.5 & 233.25 & 439.25 & 0.94 & 0.405 & 11 & 0.642 & 8.47 & 0.17 & 0.42 & Yes \\
\hline 9 & 322.8 & 617.09 & 0.91 & 0.407 & 13 & 0.550 & 8.58 & 0.18 & 0.44 & Yes \\
\hline 10.5 & 428.75 & 826.04 & 0.89 & 0.401 & 12 & 0.477 & 6.87 & 0.12 & 0.30 & Yes \\
\hline 12 & 550.55 & 1051.83 & 0.85 & 0.384 & 14 & 0.418 & 7.02 & 0.16 & 0.42 & Yes \\
\hline 13.5 & 687.71 & 1321.29 & 0.825 & 0.377 & 15 & 0.377 & 6.73 & 0.13 & 0.34 & Yes \\
\hline 15 & 844.46 & 1625.04 & 0.754 & 0.37 & 15 & 0.338 & 6.08 & 0.12 & 0.32 & Yes \\
\hline
\end{tabular}

Table15:-FOS by Seed and Idriss

\begin{tabular}{|c|c|c|c|c|c|c|c|c|c|}
\hline Depth & $\left.\sigma_{\mathrm{V}}^{\prime} \mathrm{kN} / \mathrm{m}^{2}\right)$ & $\left.\sigma_{V} \mathrm{kN} / \mathrm{m}^{2}\right)$ & $\mathrm{r}_{\mathrm{d}}$ & CSR & $\mathrm{N}_{60}$ & $\left(N_{1}\right)_{60}$ & CRR & F0S & Liquefaction \\
\hline 1.5 & 28.8 & 28.8 & 0.98 & 0.23 & 3 & 5.63 & 0.11 & 0.48 & Yes \\
\hline 3 & 57.39 & 86.82 & 0.97 & 0.45 & 5 & 7.67 & 0.15 & 0.33 & Yes \\
\hline 4.5 & 100.68 & 174.25 & 0.97 & 0.69 & 8 & 10.62 & 0.2 & 0.30 & Yes \\
\hline 6 & 159.3 & 291.73 & 0.95 & 0.90 & 12 & 14.22 & 0.24 & 0.26 & Yes \\
\hline 7.5 & 233.25 & 439.25 & 0.94 & 1.11 & 11 & 11.87 & 0.22 & 0.19 & Yes \\
\hline 9 & 322.8 & 617.09 & 0.93 & 1.31 & 13 & 12.96 & 0.24 & 0.18 & Yes \\
\hline 10.5 & 428.75 & 826.04 & 0.92 & 1.51 & 12 & 11.16 & 0.17 & 0.11 & Yes \\
\hline 12 & 550.55 & 1051.83 & 0.90 & 1.69 & 14 & 12.25 & 0.21 & 0.12 & Yes \\
\hline 13.5 & 687.71 & 1321.29 & 0.89 & 1.88 & 15 & 12.43 & 0.18 & 0.09 & Yes \\
\hline 15 & 844.46 & 1625.04 & 0.88 & 2.06 & 15 & 11.82 & 2.06 & 0.08 & Yes \\
\hline
\end{tabular}

Table1 6:-FOS by IS Code Procedure

\section{COMPARISION}

Comparing the results of 8 boreholes obtained from both the methods. 
Published Online December 2020 in IJEAST (http://www.ijeast.com)

A. Borehole I

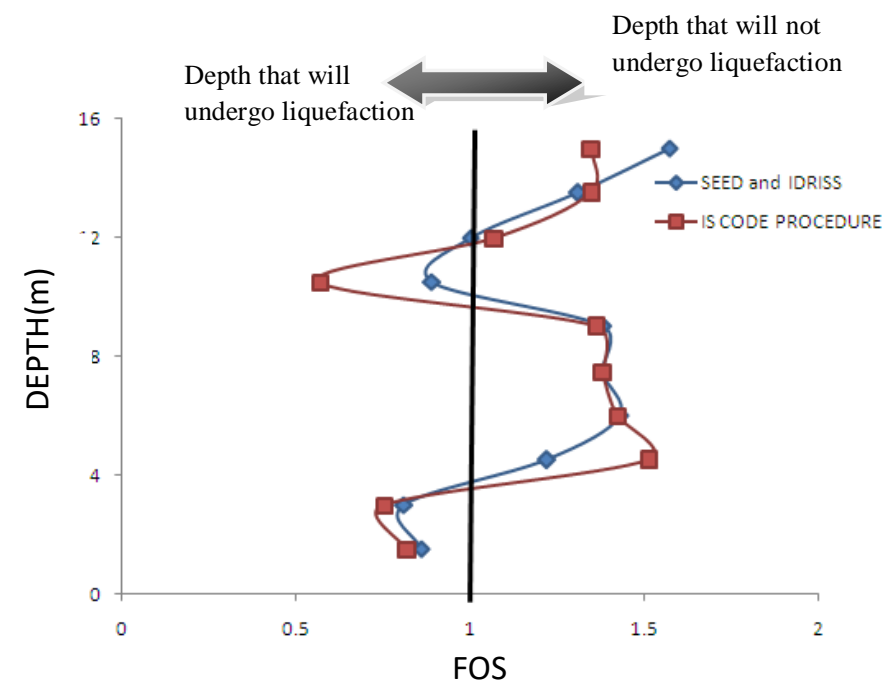

Fig 7:- FOS vs Depth

B. Borehole II

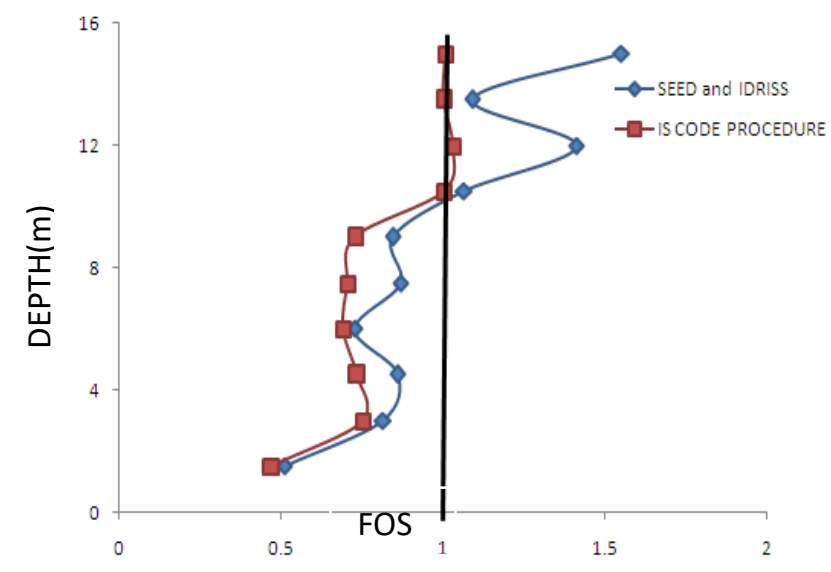

Fig 8:- FOS vs Depth

\section{C.Borehole III}

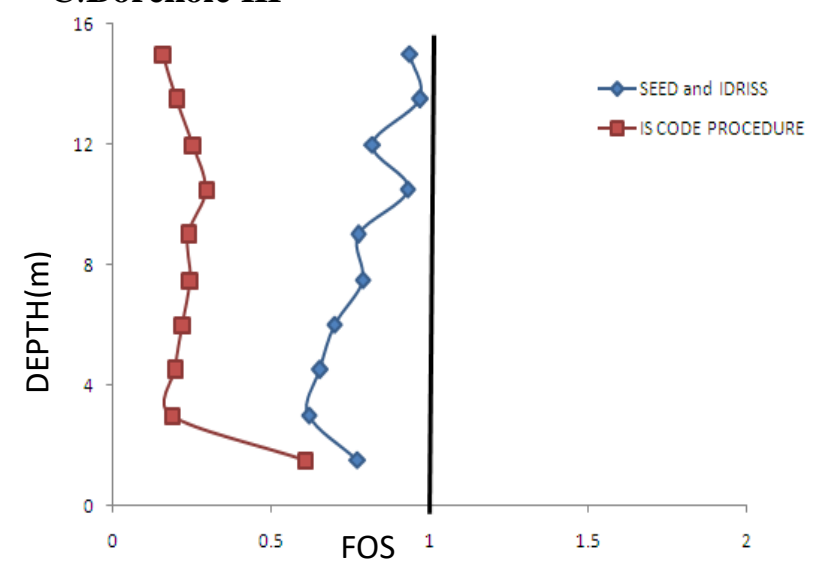

C. Borehole IV

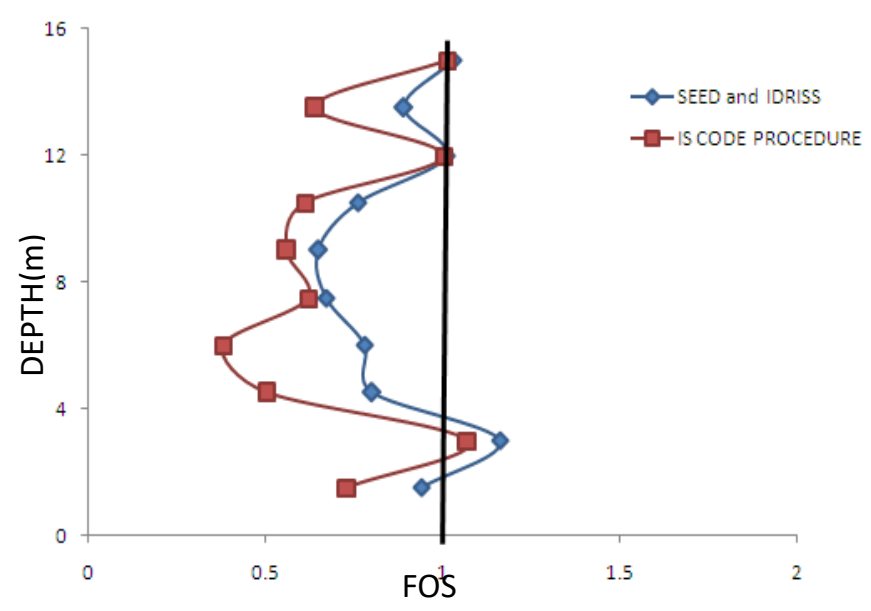

Fig 10:- FOS vs Depth

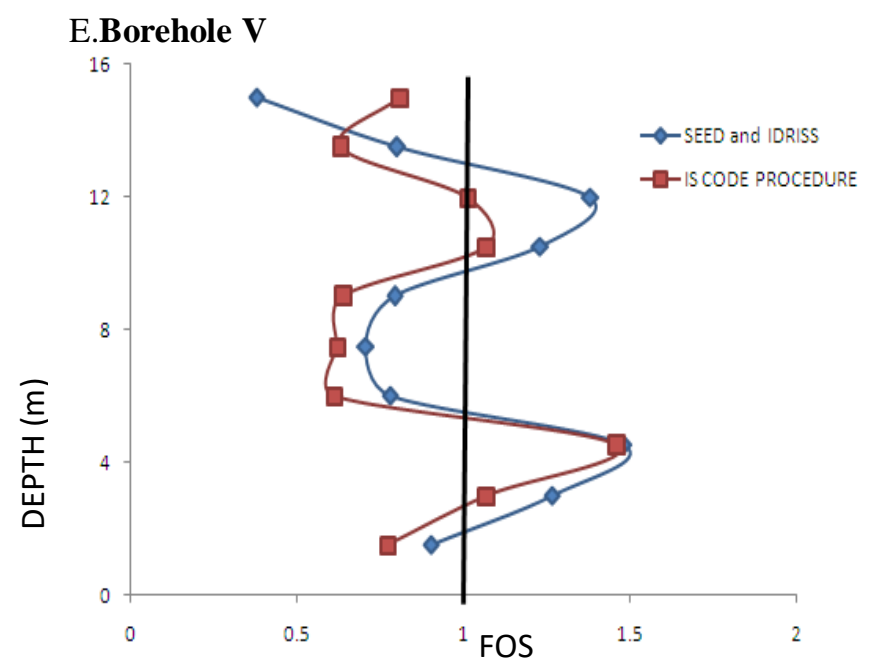

Fig 11:- FOS vs Depth

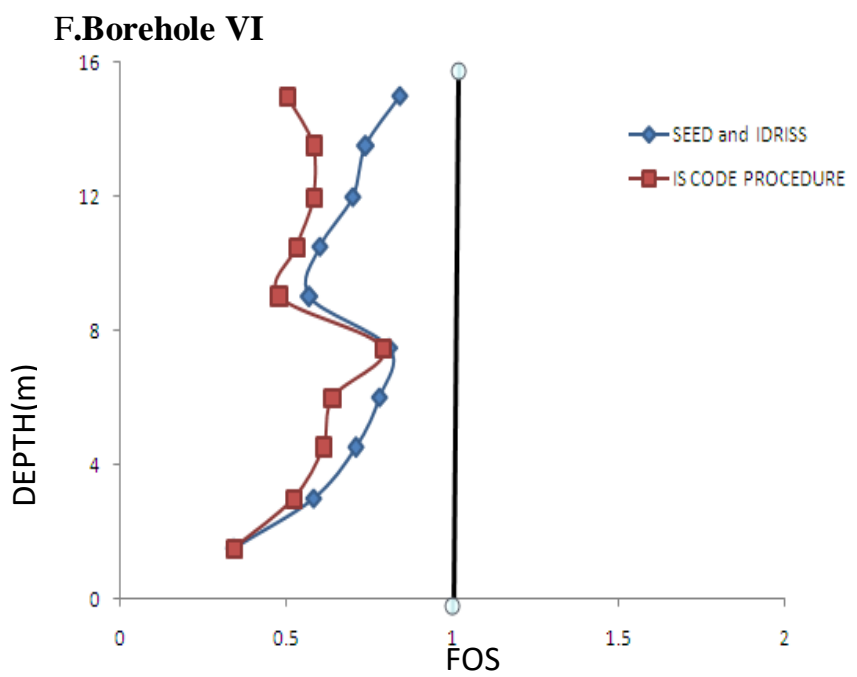

Fig 12:- FOS vs Depth

G. Borehole VII 


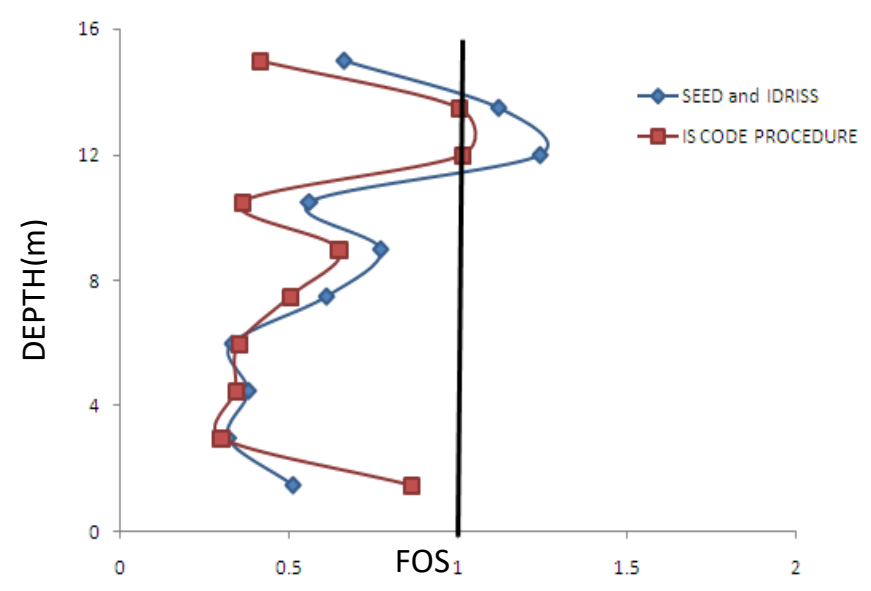

Fig 13:- FOS vs Depth

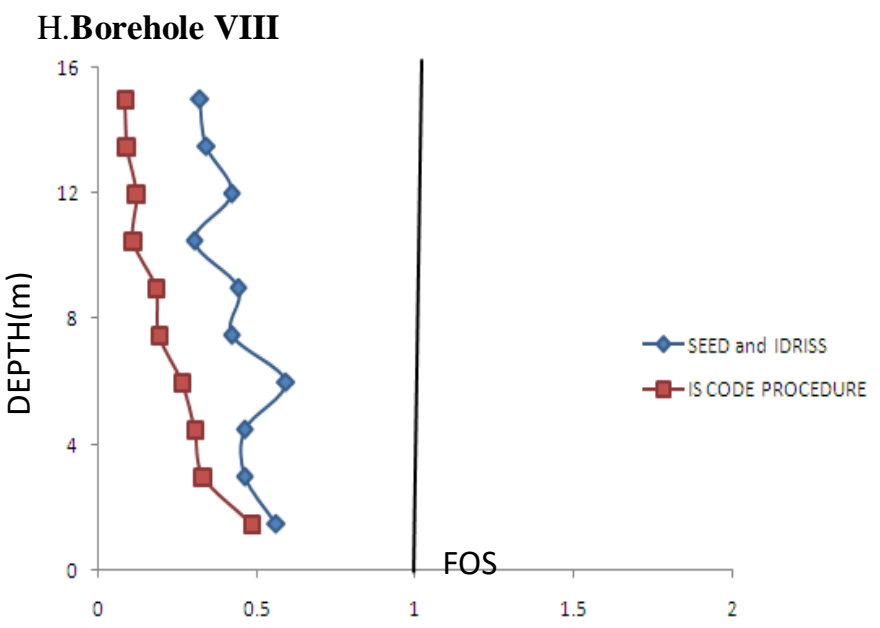

Fig 14:- FOS vs Depth

\section{RESULTS}

As per IS code 1893 (2002) Guwahati lies in Zone V. The peak ground acceleration for Guwahati is $0.36 \mathrm{~g}$ for an 8.1 magnitude earthquake. The factor of safety against liquefaction is determined for all the 95 boreholes using Seed and Idriss and IS Code Procedure. Out of the 95 boreholes 59 boreholes was found to be susceptible to liquefaction by both the methods. The lowest factor of safety against liquefaction among the layers is considered to be the factor of safety for that borehole.

From the calculation it is observed that areas like Chandmari, zoo road, Sixmile, Beltola, Dispur, G.S. Road, Uzanbazar, jalukbari and Bharalamukh are most susceptible to liquefaction.

Although the factor of safety are calculated for all 95 boreholes but tables 1 to 16 shows factor of safety withdepth of only 8 boreholes for both the methods. A comparison of factor of safety between the two methods is shownin figs 7 to 14. It is observed that for all the 95 boreholes both the methods gives almost the same results. Hence the IS code procedure may be preferred as it is a much simpler method as compared to Seed \& Idriss for evaluation of liquefaction potential.

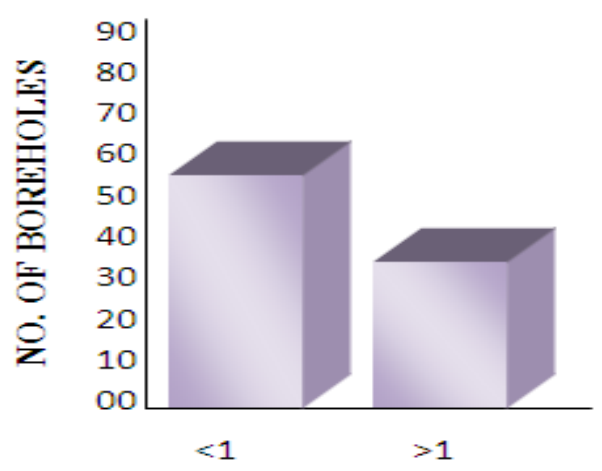

FACTOR OF SAFETY

Fig 15:- Bar diagram representing factor of safety against the number of boreholes

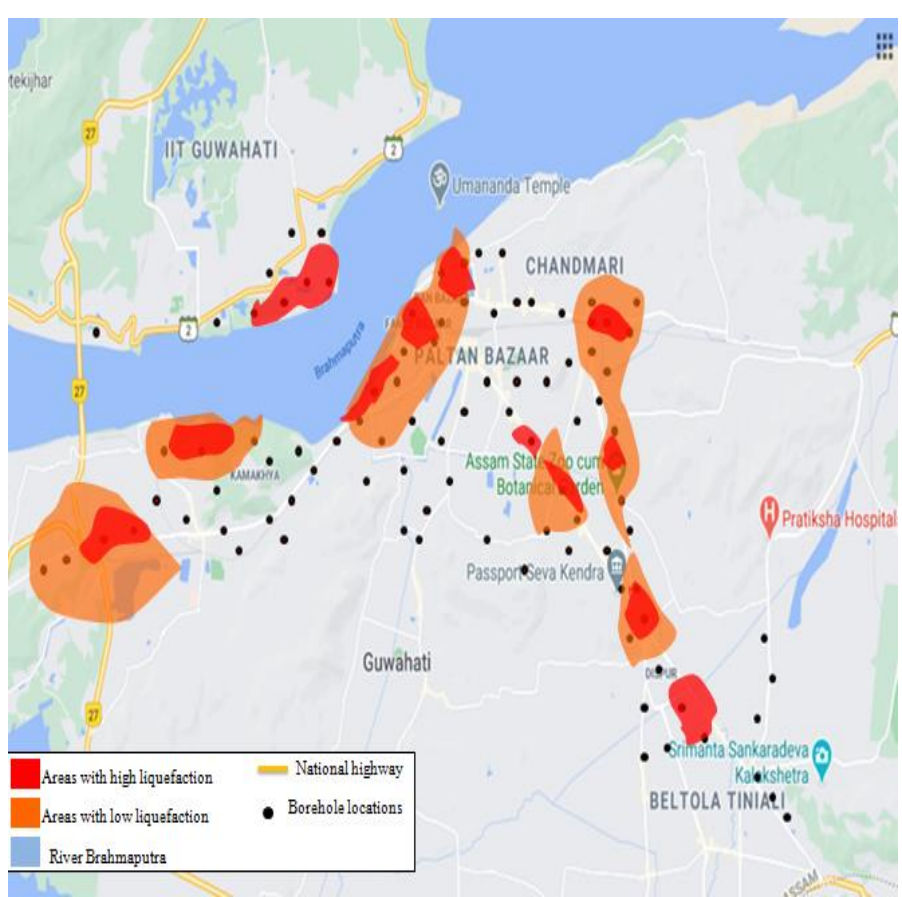

Fig 16:- Liquefaction potential map of GUWAHATI city.

\section{CONCLUSION}

In this paper the liquefaction potential of Guwahati city is assessed with the help of Seed and Idriss method (1971) and IS Code Procedure (2016). The factor of safety is calculated using 95 borelog datas, and it is found that the factor of safety is less than 1.0 for 59 boreholes. It is also observed that the total thickness of soil up to $15 \mathrm{~m}$ is susceptible to liquefaction, which means that Guwahati city area is most vulnerable to liquefaction related hazards during future earthquakes of magnitude more than 8 . From the map it is observed that the areas areas like Chandmari, Zoo Road, Sixmile, Beltola, Dispur, G.S. Road, Uzanbazar, Jalukbari and Bharalamukh 


\section{International Journal of Engineering Applied Sciences and Technology, 2020 \\ Vol. 5, Issue 8, ISSN No. 2455-2143, Pages 219-228 \\ Published Online December 2020 in IJEAST (http://www.ijeast.com)}

are most susceptible to liquefaction. Both the northern and southern bank of the city is susceptible to liquefaction.

Also, a relative comparison of factor of safety using Seed \& Idriss and IS Code Procedure method is also made.

Thus it is hoped that this paper contributes in making a broader microzonation map of Guwahati.

\section{REFERENCE}

1 Boulanger, R. W. (2003). High overburden stress effects in liquefaction analyses, J. Geotechnical and Geo-environmental Eng., ASCE 129(12), 1071082.

2 Boulanger, R. W. and Idriss, I. M. (2004). State normalization of penetration resistances and the effect of overburden stress on liquefaction resistance, in Proceedings, 11th International Conference on Soil Dynamics and Earthquake Engineering, and 3rd International Conference on Earthquake Geotechnical Engineering, D. Doolin et al., eds., Stallion Press, Vol. 2, pp. 484-91.

3 Boulanger, R. W., Mejia, L. H., and Idriss, I. M. (1997). Liquefaction at Moss Landing during LomaPrieta earthquake, J. Geotechnical and Geoenvironmental Eng, ASCE 123(5), 453-67

4 Bureau Of Indian Standards(1970) Classification And Identification Of Soil For General Engineering Purposes, IS 1498, New Delhi, India

5 Ginvinda-raju and Bhattacharya, 2011; Ranjan, 2005; Nath et al., 2008; Site specific earthquake response study of hazard assessment in Kolkata city, India. Journal of Natural Hazards. DOI 10.1007/s11069-011-9940-3.

6 Idriss, I. M. (1999). An update to the Seed-Idriss simplified procedure for evaluating liquefaction potential, in Proceedings, TRB Workshop on New Approaches to Liquefaction, Publication No. FHWARD- 99-165, Federal Highway Administration, January.

7 Idriss, I. M., and Boulanger, R. W. (2003). Relating $K$ to SPT blow count and to CPT tip resistance for use in evaluating liquefaction potential, in Proceedings of the 2003 Dam Safety Conference, ASDSO, September 7-10, Minneapolis, MN.

8 Idriss, I. M., and Boulanger, R. W. (2004). Semiempirical procedures for evaluating liquefaction potential during earthquakes, in Proceedings, 11th International Conference on Soil Dynamics and Earthquake Engineering, and 3rd International Conference on Earthquake Geotechnical Engineering, D.Doolin et al., eds., Stallion Press, Vol. 1, pp. 32-56.

9 Idriss, I. M., and Boulanger, R. W. (2006). Semiempirical procedures for evaluating liquefaction potential during earthquakes, J. Soil Dynamics and Earthquake Eng. 26, 115-30.

10 Idriss, I. M., and Boulanger, R. W. (2008).Soil liquefaction during earthquakes. Monograph MNO-
12, Earthquake Engineering Research Institute, Oakland, CA, $261 \mathrm{pp}$.

11 Iwasaki, T. (1986). "Soil liquefaction studies in Japan: state-of-the-art." Soil Dynamics and Earthquake Engineering, 5(1), 2-68.

12 Iwasaki, T., Arakawa, T., and Tokida, K. (1984). "Simplified procedures for assessing soil liquefaction during earthquakes." Soil Dynamics and Earthquake Engineering, 3(1), 49-59.

13 Iwasaki, T., and Tokida, K. (1980). "Studies on soil liquefaction observed during the Miyagi-ken Okiearthquake of June 12, 1978." Seventh World Conference on Earthquake Engineering, Istanbul, Turkey,Sept 8-13, 1-8.

14 Kramer, S.L. (1996). Geotechnical Earthquake Engineering. Prentice Hall, New Jersey (NJ), 653.

15 Nath et al. (2008) analysed Guwahati city.

16 Punmia,B.C.,Jain,A.K.,\&Jain,A.K.(2017)Soil Mechanics and Foundations.

17 Raghukanth, S.T.G and Dash S.K. (2010). Deterministic seismic scenarios for Northeast India. Journal of Seismology, 14pp.143-167.

18 Saran,Swami. (1999) Soil dynamics and machine foundation

19 Sahib Ahmed et al.(2015), conducts the standard penetration test at Lucknow

20 Seed and Idriss(1971); Seed and Idriss(1982); and Seed et al.(1985); Liao and Whitman(1986), Idriss(1999); Toprak et al. (1999); Juang et al., (2002); Cetin et al. (2004), Idriss and Boulanger(2004, 2006), determination of liquefaction potential using the deterministic as well as probabilistic approach.

21 Seed and Idriss (1982) and Seed et al. (1985), by Liao and Whitman (1986), by Idriss (1999), NCEER Workshops- (1996 and 1997), by Toprak et al. (1999), by Juang et al. (2002), by Cetin et al. (2004), by Idriss and Boulanger (2004, 2006)etc determined liquefaction.

22 Selig and Chang (1981) and Robertson (1994), cyclic liquefaction.

23 Sharma, B. and Rahaman, S.K., (2016). Use of GIS based maps for preliminary assessment of subsoil Of Guwahati City. Journal of Geoscience and Environment protection, 2016, 4, pp.106-116. 